plates coated in gold and separated by a spacer a few micrometres thick at the top (Fig. 1).

Hinds et al. measured the effect of different cavity spacings on the transmission of atoms through the gap by detecting atoms passing through the gap at different heights. Very efficient detection was achieved using a laser beam to ionize the emerging atoms. The physical separation of the gold surfaces at different heights was determined to within 5 nanometres by using monochromatic light to produce interference fringes the number of half wavelengths at a given height was found by counting the number of fringes from the bottom where the surfaces were in contact. But the effective width of the gap for trans-

\title{
MICROBIOLOGY
}

\section{Giants among the prokaryotes}

\section{Mitche/l L. Sogin}

IMAGINE a world in which prokaryotes dwarf typical eukaryotes. A glimpse of such a world is provided on page 239 of this issue ${ }^{1}$, where Angert and colleagues describe molecular data showing that symbionts that live in the gut of the surgeonfish, and which are some $80 \times$ 600 micrometres $(\mu \mathrm{m})$ in size, are closely related to Gram-positive eubacteria. This radical departure from 'normal' prokaryotic cell architecture underscores the point that many microorganisms that cannot be cultured in the laboratory may be stranger and more diverse than we realize.

Because of its enormous size, the surgeonfish symbiont Epulopiscium fishelsoni was originally described as a protist of unknown phylogenetic affinity $^{2}$. Then evidence that it is in fact a prokaryote of some sort came from electron microscopy studies ${ }^{3}$. Instead of nuclei, these giant symbionts contain bacterial-type nucleoids with no hint of surrounding membrane structure; and their flagella are bacterial-type rather than the classic $9+2$ microtubule structures found in eukaryotic cilia. So gross morphology and ultrastructure point to different conclusions about the phylogenetic history of the organism, resolution of which is unlikely to be achieved by comparative studies of physiology and biochemistry because $E$. fishelsoni (as well as most symbionts and an untold variety of microbial species) cannot be propagated in the laboratory.

Modern molecular studies ${ }^{4}$ circumvent this difficulty by using standard cloning or polymerase chain reaction (PCR) methods to isolate genes that represent evolutionary homologues from natural populations. Comparisons with a large mission of atoms is smaller than the actual size because atoms near the walls are deflected. This has a significant effect on the fraction of transmitted atoms when the cavity spacing is around $1 \mu \mathrm{m}$. The reciprocal of the transmission of the gap, the opacity (see Fig. 2), shows clearly that there is an interaction between the atom beam and the plates, and the observations agree well with the behaviour expected for the CasimirPolder force - there were no free parameters in this fitting. Sukenik et al., it seems, have succeeded in bringing nothing into this world.

C. J. Foot is in the Clarendon Laboratory, University of Oxford, Parks Road, Oxford OX1 3PU, UK. or the presence of large liquid vacuoles.

The $80 \times 600-\mu \mathrm{m}$ cell dimensions of E. fishelsoni are far greater than the examples cited above, so the theoretical constraints on cell size imposed by prokaryotic cell architecture and apparent absence of intracellular vesicular transport mechanisms will have to be reexamined. Clements and Bullivant ${ }^{3}$ proposed that the peripheral layer of highly convoluted (plasma) membranes in $E$. fishelsoni solves the problem of transport to the interior and provides a large compartment for pooling protons to feed the battery of flagella required for motility. Mechanisms for faithful distribution of nucleoid structures (containing bacterial chromosomes) to daughter cells remain unclear.

Perceived size constraints for prokaryotes, and hence interpretations of microfossils according to size, have dominated traditional thinking about the evolutionary history of eukaryotes ${ }^{9}$. The evolution of large size is considered to be an automatic consequence of the evolution of the most fundamental eukaryotic characters, the endomembrane system and the cytoskeleton. The absence of large cells from the fossil record before $1.5-2.0$ billion years ago is taken as evidence for the more recent appearance of cells with nuclei. But it now becomes clear that the assignment of eukaryotic status to microfossils is strictly an operational definition. The existence of small eukaryotes such as Nanochlorum and the discovery of giant prokaryotes lacking cytoskeletons and known vesicular transport system raises suspicions about the phylogenetic significance of size differences between eukaryotes and prokaryotes. This in turn will force reexamination of the fossil record as it pertains to the evolutionary origins of eukaryotes - indeed, molecular data ${ }^{10}$ in the form of rRNA sequence similarities are consistent with eukaryotic lineages diverging before three billion years ago.

Now, consider some of the ideas that are affected by this humbling discovery of prokaryotic giants. Size is a frequently cited (but incorrect) criterion for differentiating between eukaryotes and prokaryotes ${ }^{6}$. Assumptions such as these colour our understanding of prokaryotic physiology and dominate theories about evolution into the primary lines of descent. Although cell volumes of eukaryotes are typically $100-1,000$ times greater than those of prokaryotes, their range of sizes overlaps. For example, the chlorophyte Nanochlorum eukaryotum has a mitochondrion, a chloroplast and a nucleus contained within a cell $1-2 \mu \mathrm{m}$ in size. Very long but skinny bacteria (greater than $200 \mu \mathrm{m}$ by less than 0.75 $8.0 \mu \mathrm{m}$ ) have been described ${ }^{7,8}$, but they contain only nominal amounts of cytoplasm because of their spiral morphology
Mitchell L. Sogin is at the Center for Molecular Evolution, Marine Biological Laboratory, Woods Hole, Massachusetts 02543, USA.

\footnotetext{
1. Angert, E. R., Clements, K. D. \& Pace, N. R. Nature 362, 239-241 (1993).

2. Fishelson, L., Montgomery, W. L. \& Myrberg, A. A. Jr Science 229, 49-51 (1985).

3. Clements, K. D. \& Bullivant, S. J. Bact. 173, 5359 5362 (1991).

4. Delong, E. F. Proc. natn. Acad. Sci. U.S.A. 89, 56855689 (1992).

5. Olsen, G. J. et al. Nucleic Acids Res. 20, 2199-2200 (1992).

6. Cavalier-Smith, T. Symp. Soc. Gen. Microbiol. 32, 33-84 (1981).

7. Blakemore, R. P. \& Canale-Parola, E. Arch. Mikrobiol. 89, 273-289 (1973).
8lakemore, R. P. \& Can

8. Demoulin, V. \& Janssen, M. P. Phycol. J. 16, 55-58 (1981).

9. Schopf, J. W. \& Oehler, D. Z. Science 193, 47-49 (1976)

10. Sogin, M. L. Curr. Opin. Genet. Devel. 1, 457-463 (1991).
} 